\title{
Design of Intelligent Classroom Bell System Based on Internet of Things
}

\author{
Yongqi Han ${ }^{1, a}$, Xuanli Zhao ${ }^{1, b}$, Mingwei $\mathrm{Li}^{1, \mathrm{c}}$ and Yun Zhang ${ }^{2, \mathrm{~d}}$ \\ ${ }^{1}$ Jilin Agricultural University College of Information Technology, Changchun,130118, China \\ ${ }^{2}$ Changchun University of Science and Technology College of Information \\ Engineering,Changchun,130600,China \\ a yongqihan@163.com, b 1119696606@qq.com, c 652694996@qq.com, d 250814909@qq.com
}

Keywords: intelligent ringing; ZigBee; Internet of things; Java serial communication

\begin{abstract}
This document focuses on networking technology, ZigBee technology and sensor technology and PC serial communication technology to complete the classroom bell system based on a ZigBee networking design.The system has the advantage of humanization, intelligent, in addition to the completion of the basic functions of the bell, with the functions of integrated fire control and security features, you can achieve more intelligent modern teaching management.

Through this system, management can be timely for each classroom teaching,and it also can be to a single classroom or more classroom immediately or timing voice broadcast.Broadcast content can be pre-recorded speech, such as the commonly used on school-related speech, test-related speech, broadcast or directly enter the text, and it can also be MP3. Data docking with the educational system of the course information, to achieve the daily tasks on a school bell, the whole process can be carried out in without manual intervention, to save more manpower and resources.
\end{abstract}

\section{Introduction}

The teaching building is the main places of learning for every teachers and students in school , the existing management model obsolete equipment, aging, it is difficult to meet the requirement of modern teaching. The bell of the teaching building just is set in the fixed place, unified time bell sound, smaller, more people or in the distance of the circumstances, you can not hear the bell ring, affecting the normal teaching, and the bell single. In the examination process, the existing ring cannot broadcast notice before the exam, test for attention, each examination room curls time from each examination room by teacher according to the respective time arrangement, unable to achieve synchronization. In the examination process, and if changes are made to the papers, you will also need a separate notice for each classroom of the candidates, less efficient.

In order to improve the teaching efficiency, more humanized service of teachers and students, we developed intelligent bell system based on Internet of things. This system can provide a variety of ring tones, can be any of a class of the day on the bell ringing can be set, speech or music MP3. Each classroom has a terminal, timely inform the teacher started the class; examination management system, it will issue instructions for each step exam, exam candidates can play notes, the end of the exam to make exams coming to an end as reminder, may carry on the notice of one or more test in the test, greatly improving the examination efficiency. In order to deal with the emergency, the system set up emergency voice commands, do emergency play immediately notice, guide the orderly evacuation of teachers and students, to reduce casualties.

\section{System Architecture}

The whole system is divided into three parts: Management of the control terminal;The coordinator; Terminal node Installed in the classroom.

Management of the control terminal: Using C/S structure with Java RCP graphic technology development of the management software, software and external Coordinator on the computer communication, the coordinator through ZigBee wireless network technology transfer command, to control each node of the classroom. In order to prevent the network transmission delay large-scale 
system, using early commands to each node one by one to send ready, send broadcast start command in time arrived, so as to achieve the large-scale network without delay.

The coordinator in the design of the JN5148-001-M04 minimum system constituted by module, peripheral includes RS232 interface communication with the host computer, used for LED power indicator, network, operation status display, a watchdog circuit and power supply circuit.

The classroom terminal node: assign a different address of 16 bits each classroom terminal node, its hardware part consists of ZigBee wireless microcontroller, sensor, JN5148-001-M04 6658 speech synthesis module, a music playing module. The block diagram shown in Fig.1.

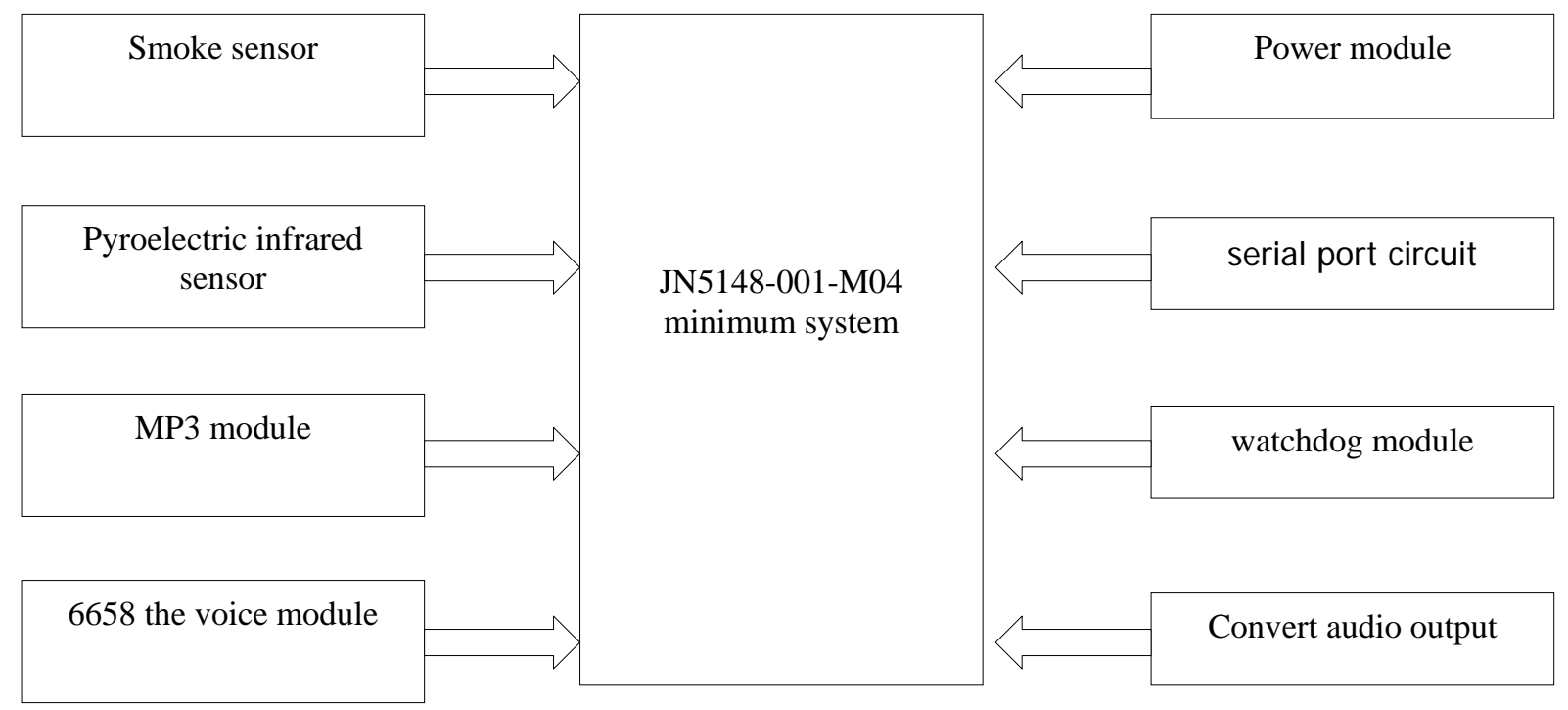

Fig.1. JN5148-001-M04 minimum system

\section{Design of the Coordinator}

The coordinator is the core of ZigBee wireless sensor network, to assume the task of the establishment and management of wireless sensor network, collecting and processing information, upload the data of each node to other nodes to send control command, communications.

Working voltage JN5148-001-M04 module for 3.3V, DC-DC step-down circuit power supply part adopts LM1117-3.3 regulator chip consists of the supply voltage of $5 \mathrm{~V}$ or $6 \mathrm{~V}$ into $3.3 \mathrm{~V}$ in order to ensure the normal work of the LED coordinator; indicating circuit comprises a power supply indicating lamp module, normal work indicating lamp, group net success indicator; independent watchdog circuit of watchdog circuit is composed of a ADM706 chip, if the watchdog input in 1.6 seconds without triggering, the circuit will start.

\section{Design of the terminal node}

\section{The design of main control module}

ZigBee wireless microcontroller choice from the actual application demand angle, in view of the system on the stability and transmission distance considerations. The JN5148-001-M04 module of the design by the British Jennic company recently launched a high emission power. JN5148 is the latest Jennic launched the third generation of ultra low power consumption, low cost wireless microcontroller, the integrated control processor and RF circuit in one, using JN5148-001-M04 module in node development to avoid complex design of RF unit, but also in the complex processor performance, low power consumption, on-chip resource, the development difficulty the extent and so on, compared with other ZigBee module JN5148 has irreplaceable advantages of ${ }^{[1]}$. The system diagram as shown in Fig.2. 


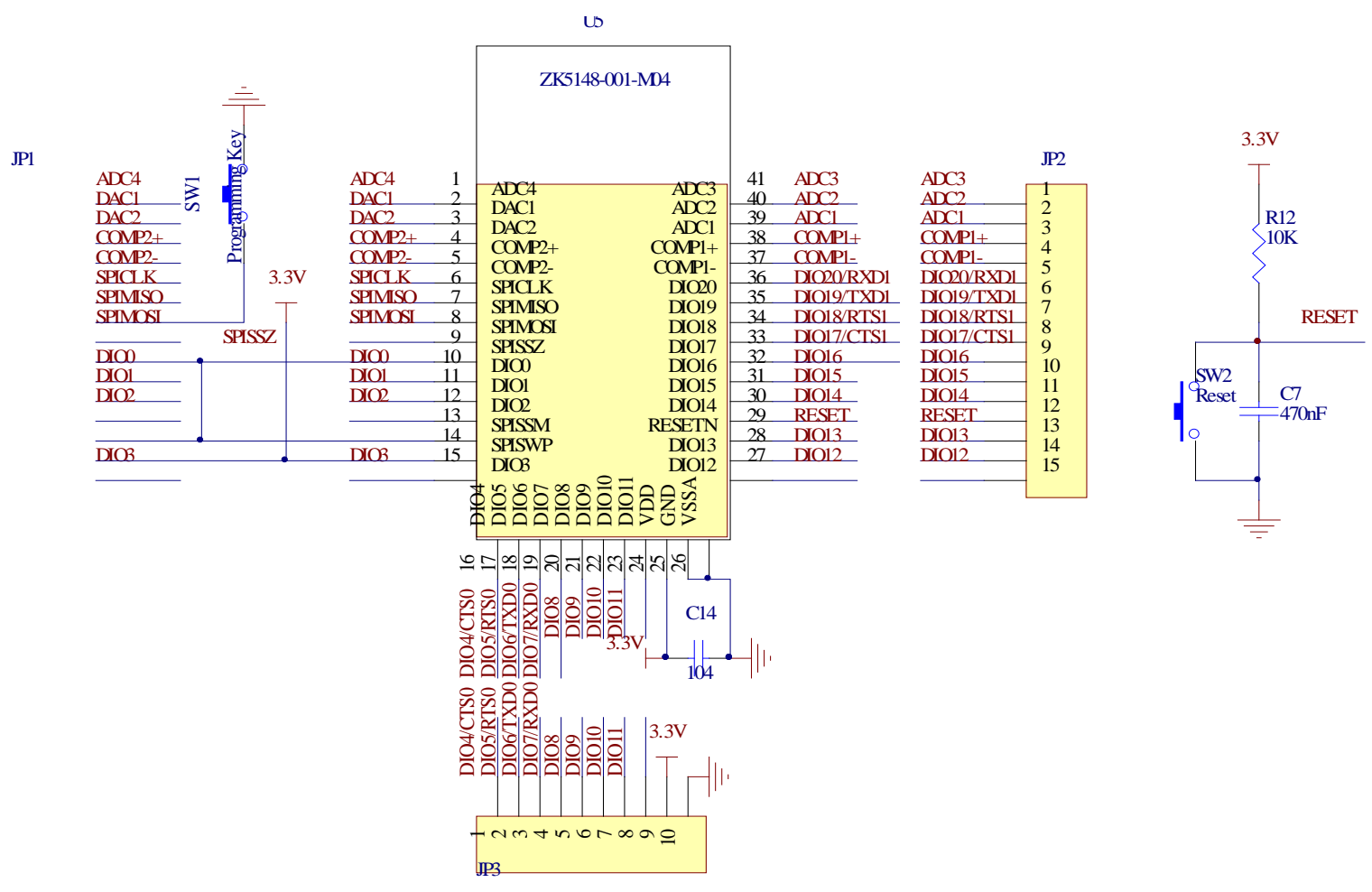

Fig.2 System diagram of JN5148 main control chip

This module uses the Jennic JN5148 wireless micro controller provides a comprehensive solution, with large capacity memory, CPU and radio performance is high, its contain RF components. The interior consists of a 32 bit RISC structure of CPU, with 128KB ROM and 128KB RAM, also provides the 513KB Flash used to store programs and data. JN5148 series module hardware resources are rich, including up to 21 DIO, 4 ADC 12 bit, 2 DAC 12 bit, 2 comparators, 3 Timer, 2 UART, SPI interface, its internal structure diagram as shown in figure 3-1. The user only needs to develop and manufacture and module connected with the power supply and peripheral devices, such as switches, actuator and sensor, which greatly simplifies the development process of product .

\section{Design of MP3 music player module}

This is the MP380 music playback module, via the TF storage playback of audio files with a song and a song and volume and the volume reduction and suspension of five control terminal.

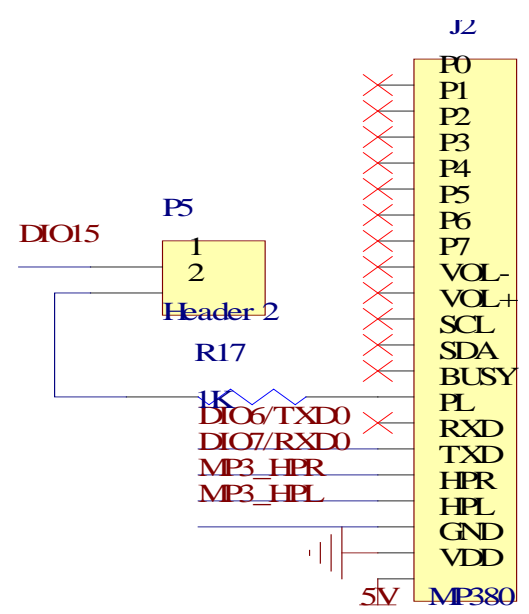

Fig. 3 MP3 circuit

Design and synthesis of module 6658 speech

Effect of speech synthesis module 6658 is the text data has become a voice in the play out through the sound. Text 6658 disposable most can play 4k. 


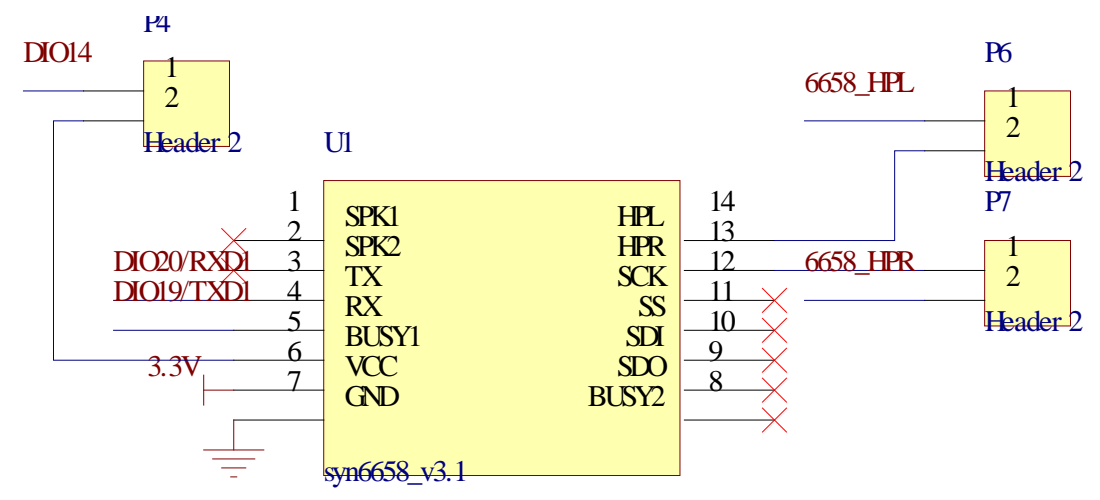

\section{The sensor module}

Fig.4 syn6658 circuit

The sensor node consists of routing node and terminal node.The sensor node is designed in JN5148-001-M04 to achieve the minimum system module based on.To join the system required MP3 modules and TF card,in the JN5148-001-M04 minimum system module 6658 module,W25Q32 flash ROM and the smoke spread the classroom environment,constitutes a sensor node.

\section{Summary}

Compared to traditional mode, this system more humanized, modernization, the system only needs a PC machine, management to the entire campus bell equipment,security design system make the teaching set equipped with more safe,effective to ensure the safety of property,and when the voice broadcast function can make you more convenient for broadcasting notice.Although at the beginning of the design consideration of many,but still encounter many problems in the actual implementation, such as a large number of personnel flow will have an impact on the ZigBee network,the network at the same time a large number of data transmission will be the loss of data packets at a certain moment,the next operation,return efficiency value when we will further improve the PC processing, in order to reduce the corresponding time of upper computer, lower computer we will increase in signal intensity, to reduce the flow of personnel of the signal interference, allowing the system to run more stable.

\section{Acknowledgments}

This work was funded by the Youth Foundation of Jilin Agricultural University under Grant No. 201326.2015 national college students innovation and entrepreneurship training program,2015 Jilin college students innovation and entrepreneurship training program,Multifunctional Family Assistant.The Youth Foundation of Changchun University of Science and Technology, based on cloud computing multimedia database research (2014). The 12th five-year plan subject of jilin province department of education: Study and Practice of Private Schools school-enterprise cooperation computer science engineer culture of excellence.

\section{References}

[1] Dai Chengbin. Research of ZigBee low energy consumption and high reliability, Guangdong: Guangdong University of Technology(2014)

[2] Pang Na. Research of ZigBee mesh network for wireless sensors, Jilin: Jilin University(2010)

[3] Nielin WCF service (the SerialPort class implements the serial communication), referenced in the web program.

[4] Daniele Miorandi, Sabrina Sicari, Francesco De Pellegrini. Internet of things: Vision, applications and research challenges. Ad Hoc Networks, 10 (2012) P1497 1516

[5] JN-UG-3064-JN5148-SDK-2v0 [EB/OL]. http://www.nxp.com/jennic2010. 